\title{
Using Transformations to Predict and Smooth Time Series
}

\section{${ }^{1}$ Jassim N Hussain, ${ }^{2}$ Zainab Hassan Radhy, ${ }^{3}$ Sackineh shamel jasem}

Jasim.nasir@uokerbala.edu.iq

${ }^{1}$ University of Kerbala / Administration and Economics College / Department of Statistics

Zainb.hassan@qu.edu.iq

${ }^{2}$ College of computer science and information technology - University of Al-Qadisiya - Iraq

${ }^{3}$ University of Wasit / Administration and Economics College / Department of Statistics

Sshamil@uowasit.edu.iq.

Article History: Received:11 January 2021; Accepted: 27 February 2021; Published online: 5 April 2021

Abstract- Time series has a leading position in statistical Analysis. Nowadays, many economic and industrial operations have been built based on time series. These operations include predicting the product demand variation, the future product prices oscillation, the stock storing control etc. This paper presents a study to show the effect of transformation and smoothing on the performance of the time series. The research results have shown a significant improvement in time-series operation can be noticed when the principles of transformation and smoothing are applied on time series.

\section{Introduction}

Time series topic is one of the essential topics nowadays because it is starting to be applied to different types of science widely. Mathematical-statistical possesses in analyzing the time series have begun to provide important functions of estimation. Furthermore, other involved significant points have made important decisions, and they used to simulate some mathematics and statistics samples for the given problem. Time series use parameters to predict the future and make the right resolution for stable series.

Stationary of series is very important in Analysis because it provides a stable mathematical model of series. Smoothing models may be classified into two categories. The first is which contains constant parametric and the second is of variable parameters, median and variance. The constant parametric model has main ways to solve because its parameters are stationary for each domain of data, i.e. its work on making series stationery by making differences and mathematics relating such as logarithms transformations and square roots etc.

Making transformations is very important in time series analysis because it makes data prepared to analyze and more accurate to get the estimation function. In this research, the transformation will be used to make a function stationary then getting smoothing function. From another view, methods of smooth ratios may deal with variables and states that, difficult to deal with it because parametric estimations smooth in a matter that easy to solve.

There are many ways to use these methods in time series analyzing, which working on smoothing data before applying statistic samples on it and deal with the varying in parameters. So, this paper searches for sample preferences and if smoothing methods treated it, then compared it with the ARIMA model.

\section{Theatrical introduction}

The time series is a set of observations for a specific phenomenon during a time period. Mathematically, it is a sequence of random variables defined over a probability space of multiple parameters and indicated by the index $t$, which is an item of $\mathrm{T}$. Time series usually written as $\{x(t) ; t \in T\}$ and it consists of two variables. One of them varies with time, and the others are dependent $(y=f(x))$.

\section{Changes which affect the improvement of time series}

Any time series is affected by multiple factors natural, economic, seasonal etc., and that effects on the general structure of time series even in long or short ranges. Figure 1 shows the seasonal variables which are iterative changes in year seasons. While figure 2 highlights the cyclic variables which are influencing the time series vibrations and repeated every regular period, it may occur every 1 to 10 years. In random variable time series, there 
are some changes that occur suddenly in data which are difficult to be predicted. Such types of data are important in spite of their difficulty, but it may appear as small waves in time series data.

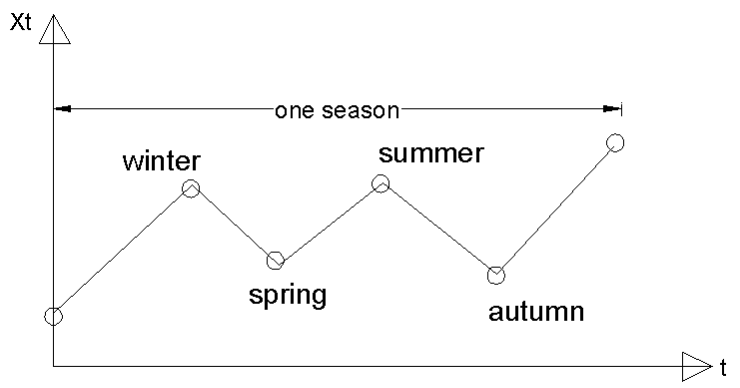

Figure 1, the seasonal time series

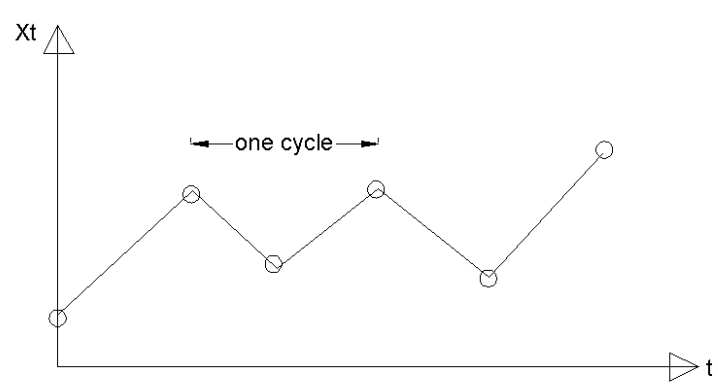

Figure 2, the cyclic variable time series

\section{Stationary time series}

Stationary and non-stationary of data are essential and important in time series analysis. Graphing time series for a period $[\mathrm{t}, \mathrm{t}+\mathrm{h}]$ may sometimes be matched with graphing the series in another interval $[\mathrm{s}, \mathrm{s}+\mathrm{h}]$ and that refers to time homogenous in series behaviour, which called stationary. This time series is further divided into two parts:

2.1. Strictly stationary

The series $\{x(t)\}$ considered as strict if the probability distribution $x_{t 1}, x_{t 2} \ldots \ldots x_{t n}$ is the same for $x_{t 1+k}, x_{t 2+k}, \ldots . x_{t n+k}$ for all selected time intervals $t_{1}, t_{2}, \ldots, t_{n}$ and for any constant. The time series said to be strict when the three previous conditions are applicable.

2.2. Weak stationary

It means the common variable probability distribution $\left(x_{t 1}, x_{t 2} \ldots \ldots x_{t n}\right)$ to some extent may be changed with time if the mean and variance were constant, and if covariance $\left(x_{t 1}, x_{t+k}\right)$ is a function to slowing down the Interval $\mathrm{K}$ and independent on time, t.

\section{Non- stationary time series}

Practically, most time series is non-stationary, and it might be impossible to prove using diagrams or statistic tests. For example, economic parametric usually considered a non-stationary time series because of its generality. For such reason, it has to be converted into stationary one to facilitate its modelling process. This series includes two cases:

\subsection{Difference Modifications}

In 1976, Box and Jenkins completely described the samples and introduced guides to understanding non-stationary series and converting it. If $\left\{x_{t 1}\right\}$ expressed a non-stationary time series. Then it can be converted using the following equation:

$$
Y_{t}=\Delta^{d} x_{t}
$$

Where $(\Delta=1-B)$ and $\mathrm{B}$ is called the indicator of back difference.

If $B_{x t}=x_{t-1}$ and $B^{2} x_{t}=x_{t-2}$, then the process of time decreasing will be suitable when the process is described by the difference. For instance, if the time series was non-stationary, then the no-stationarity can be processed so that the time series becomes stationary by using the first difference as explained in the following equation:

$$
x_{t}^{\prime}=x_{t}-x_{t-1}
$$

And by applying the process of time reflecting on the previous equation, it becomes:

$$
x_{t}^{\prime}=x_{t}-B x_{t-1}=(1-B) x_{t}
$$


It can be noticed that the first difference expressed by (1-B) and comparing with the second difference, the last equation becomes:

$$
\begin{gathered}
X_{t}^{n}=X_{t}^{\prime}-X_{t-1}^{\prime}=\left(X_{t}-X_{t-1}\right)-\left(X_{t-1}-X_{t-2}\right) \\
\Rightarrow\left(1-2 B+B^{2}\right) X_{t}=(1-B)^{2} X_{t}
\end{gathered}
$$

The purpose of taking the first and second differences is to satisfy the stationary of time series.

\subsection{The case of non-stationary variance}

It is one of the most important problems which avoids obtaining the accurate sample. But using converting as (logarithms, square roots etc.) for time

series data may fix the problem.

Converting time series may give a stationary stable one. In general, ARIMA samples and such converting introduced important functions for smoothing.

There are four available transformations for positive series; when supposed that

$Y_{t}>0$ is the main series and $x_{t}$ is converted series. The transformations are:

- Logarithmic transformation $\left(x_{t}=\ln \left(Y_{t}\right)\right)$

- Logistic transformation $x_{t}=\ln \left(\frac{C Y_{t}}{1-C Y_{t}}\right)$

Where $\mathrm{c}=\left(1-e^{-6}\right) 10^{-\operatorname{ceil}\left(\log 10\left(\max \left(Y_{t}\right)\right)\right)}$

Ceil w: is an integer number equal or larger than $w=\log 10\left(\max Y_{t}\right)$

- Square root transformation $x_{t}=\sqrt{Y_{t}}$

- Box-Cot transformation

$$
X_{t}=\left[\begin{array}{cc}
\frac{Y_{t}^{\lambda}-1}{\lambda} & ; \lambda \neq 0 \\
\ln Y_{t} & ; \lambda=0
\end{array}\right]
$$

\section{Time series models:}

It includes the unseasonal time series models types stationary and non-stationary. Following three models are presented:

1) Auto-Regressive model (AR)

Yule (1926) was the first who studied the stationary time series, while Wiker completed Yule's work and provided a general form for autoregressive samples as expressed in the equation below:

Where:

$$
X_{t}=C+\varphi_{1} X_{t-1}+\varphi_{2} X_{t-2}+\varphi_{p} X_{t-p}+a_{t}
$$

$a_{t}:$ represents the random error or noise. It is usually distributed $N\left(0, \sigma_{a}^{2}\right)$

$C$ : a constant and $-1<\varphi<1 ; \varphi_{1}, \varphi_{2}, \varphi_{3}$ represents parameters of the autoregressive sample.

The autocorrelation function gradually exponentially decreases while

, while the partial autocorrelation has been lost after the period (P). For an instant, when $\mathrm{P}=1$, the above equation becomes:

$$
X_{t}=C+\phi_{1} X_{t-1}+a_{i}
$$

2) Autoregressive- Moving average models ((ARIMA)

Slutzky and Wold participated in improving sample to three-dimension in the estimation and named it by "auto regressive -mixed moving average and autoregressive used when data are stationary. Where:

$$
X_{t}=C+\varphi_{1} X_{t-1}+\varphi_{2} X_{t-2}+\cdots+\varphi_{p} X_{t-p}+a_{t}-\theta_{1} a_{t-1}-\theta_{2} a_{t-2}-\cdots-\theta_{q} a_{t-q}
$$

The function of partial autocorrelation generally decreased, as an example, ARIMA becomes

$$
X_{t}=\varphi_{1} X_{t-1}-\theta_{1} a_{t-1}+C+a_{t}
$$


3) Autoregressive integrated moving average models

Box and Jenkins (1976) described the overall samples and affected guides to understanding and dealing with data stationery. A sample has been affected by them capable of dealing with non-stationary series and converting it into stationary one by using differences odd degrees $(\mathrm{d}=1,2)$. It can be written by ARIMA $(\mathrm{p}, \mathrm{d}, \mathrm{q})$ as:

Where:

$$
\varphi(B)(1-B)^{d} X_{t}=\theta(B) a_{t}
$$

$$
\begin{aligned}
& \varphi(B)=1-\varphi_{1} B-\varphi_{2} B^{2}-\cdots-\varphi_{p} B_{p} \\
& \theta(B)=1-\theta_{1} B-\theta_{2} B^{2}-\cdots-\theta_{p} B_{p}
\end{aligned}
$$

2. Exponential smoothing series الاسية

Pegels (1969) classified smoothing methods, which is one of the most important methods to estimate the time series and contained different ways to deal with all series types.

It is a method which smoothing the non-seasonal series and gives a pervious

$$
F_{t}=\alpha X_{t}+(1-\alpha) F_{1}
$$

\section{Single exponential smoothing method}

Provides by C.C. Holt (1958) which used non-seasonal time series and then Browns (1963) worked on using it for most time series types. Harrison (1965) provides guides to apply the method as follow:

1. Let $X_{t-N}$ are --------- and weak to be available, so at $X_{t-N}$ an approach value has to be used. Such as $F_{t+1}=F_{t}+\left(\frac{X_{t}}{N}-\frac{X_{t}-N}{N}\right)$

$\mathrm{F}_{\mathrm{t}}$ will be an alternative data

$$
F_{t+1}=F_{t}+\left(\frac{X_{t}}{N}-\frac{F_{t}}{N}\right)
$$

Data obtained from the previous equation are more stationary because it depends on a weight which is a fractional value. The equation can be simplified as:

$$
F_{t+1}=\left(\frac{1}{N}\right) X_{t}+\left(1-\frac{1}{N}\right) F_{t}
$$

Smoothing $F_{t+1}$ depending on a specific ratio which is $(1 / \mathrm{N})$ for all real data found, and previous data weight depending on $\left(1-\frac{1}{N}\right)$.

$\mathrm{N}$ : is a positive number

$(1 / \mathrm{N})$ : is a value between zero and one.

If $\mathrm{N}=1$, and let $\alpha=\frac{1}{N}$

$$
F_{t+1}=\alpha X_{t}+(1-\alpha) F_{t}
$$

The previous equation is a simple exponential smoothing.

$F_{t+1}:$ smoothing statistic

$F_{t}$ : smooth value for the previous interval

$\alpha$ : smoothing series

$X_{t}$ : time series

\section{Paper Methodology}

The paper methodology is based on applying the rainfall data on the theoretical equation presented and discussed above. The first process is to draw the observations on $\mathrm{Y}$-axis and time at $\mathrm{X}$-axis.

\subsection{Matching sample of ARIMA}

To find the best match sample of time series for rainfall, two criteria have been used. They are the Aiki and MSE criteria.

1. Aiki information criteria (AIC)

$\mathrm{K}$ : number of the parameter is a sample

$$
\operatorname{AIC}(K)=n \ln \sigma_{\varepsilon}^{2}+2 k
$$

$\sigma_{\varepsilon}^{2}$ : Value of variance 
$\mathrm{N}$ : number of observations

To get the sample, choose the minimum value of AIC (k).

2. MSE

$\mathrm{N}$ : number of observations

$$
M S E=\frac{\sum_{n=1}^{n}\left(X_{t}-\widehat{X_{t}}\right)^{2}}{n-(k+1)}
$$

$\mathrm{K}$ : number of parameters

$Y_{t}$ : Value of observations

The minimum MSE; the best model used.

\section{Results and discussion}

Based on the paper methodology, the following results were obtained

1 Finding the best rainfall sample

Using ARIMA (p,d,q) and by using $\operatorname{AIC}(K)$ and MSE criteria, it has been found that (from the table (1)) sample ARIMA A $(5,0,2)$ was the best because it has the minimum value.

\begin{tabular}{|c|c|c|c|c|c|c|c|c|}
\hline Model & MSE & AIC $(\mathrm{k})$ & Model & MSE & AIC(k) & Model & MSE & AIC(k) \\
\hline ARIMA(0,0,1) & 12783 & 126.307 & ARIMA(1,0,4) & 13353 & 126.894 & ARIMA(4,0,1) & 12072 & 125.536 \\
\hline ARIMA(0,0,2) & 13062 & 126.597 & ARIMA(1,0,5) & 13831 & 127.367 & ARIMA(4,0,3) & 11015 & 124.303 \\
\hline ARIMA(0,0,3) & 13037 & 126.572 & ARIMA(2,0,1) & 12323 & 125.813 & ARIMA(4,0,4) & 11546 & 124.937 \\
\hline ARIMA(0,0,4) & 11616 & 125.018 & ARIMA(2,0,2) & 11860 & 125.298 & ARIMA(4,0,5) & 10974 & 124.253 \\
\hline ARIMA(0,0,5) & 11994 & 125.449 & ARIMA(2,0,3) & 10991 & 124.273 & ARIMA(5,0,1) & 12924 & 126.454 \\
\hline ARIMA(1,0,0) & 12728 & 126.249 & ARIMA(2,0,4) & 11624 & 125.027 & ARIMA(5,0,2) & 10673 & 123.878 \\
\hline ARIMA(2,0,0) & 13166 & 126.704 & ARIMA(2,0,5) & 12009 & 125.466 & ARIMA(5,0,3) & 11963 & 125.414 \\
\hline ARIMA(3,0,0) & 13288 & 126.828 & ARIMA(3,0,1) & 11595 & 124.994 & ARIMA(5,0,4) & 13068 & 126.604 \\
\hline ARIMA(4,0,0) & 13364 & 126.905 & ARIMA(3,0,2) & 13776 & 127.314 & & & \\
\hline ARIMA(5,0,0) & 13648 & 127.188 & ARIMA(3,0,3) & 11763 & 125.187 & & & \\
\hline ARIMA(1,0,1) & 13171 & 126.709 & ARIMA(3,0,4) & 12092 & 125.559 & & & \\
\hline ARIMA(1,0,2) & 13130 & 126.667 & ARIMA(3,0,5) & 10900 & 124.161 & & & \\
\hline ARIMA(1,0,3) & 12812 & 126.337 & & & & & & \\
\hline
\end{tabular}

2 Smoothing using transformation

Autoregressive samples and moving average samples has been applied on rainfall data after converting it by using square root method transformation. The best matching sample has been found at ARIMA $(1,0,0)$.

\begin{tabular}{|c|c|c|l|c|c|c|c|c|}
\hline Model & MSE & AIC(k) & Model & MSE & AIC(k) & Model & MSE & AIC(k) \\
\hline ARIMA(0,0,1) & 7.512 & 32.760 & ARIMA(1,0,4) & 7.804 & 41.2298 & ARIMA(4,0,1) & 7.139 & 40.1383 \\
\hline ARIMA(0,0,2) & 7.645 & 34.970 & ARIMA(1,0,5) & 8.084 & 43.6649 & ARIMA(4,0,2) & 6.432 & 40.8700 \\
\hline ARIMA(0,0,3) & 7.6 & 36.900 & ARIMA(2,0,1) & 7.177 & 36.2030 & ARIMA(4,0,3) & 6.657 & 43.2880 \\
\hline ARIMA(0,0,4) & 6.416 & 36.840 & ARIMA(2,0,2) & 6.14 & 36.3100 & ARIMA(4,0,4) & 6.765 & 45.4800 \\
\hline ARIMA(0,0,5) & 6.6 & 39.180 & ARIMA(2,0,3) & 7.773 & 41.1800 & ARIMA(4,0,5) & 7.664 & 49.0070 \\
\hline ARIMA(1,0,0) & 7.456 & 32.660 & ARIMA(2,0,4) & 7.093 & 42.0590 & ARIMA $(5,0,1)$ & 8.61 & 44.4470 \\
\hline ARIMA(2,0,0) & 7.745 & 35.130 & ARIMA(2,0,5) & 6.954 & 43.8180 & ARIMA $(5,0,2)$ & 6.65 & 43.2770 \\
\hline ARIMA(3,0,0) & 7.815 & 37.271 & ARIMA(3,0,1) & 6.792 & 37.5328 & ARIMA(5,0,3) & 6.432 & 44.8700 \\
\hline
\end{tabular}




\begin{tabular}{|c|c|c|c|c|c|c|c|c|}
\hline $\operatorname{ARIMA}(4,0,0)$ & 7.877 & 39.344 & $\operatorname{ARIMA}(3,0,2)$ & 8.708 & 42.5880 & $\operatorname{ARIMA}(5,0,4)$ & 8.917 & 50.8800 \\
\hline $\operatorname{ARIMA}(5,0,0)$ & 8.081 & 41.660 & $\operatorname{ARIMA}(3,0,3)$ & 6.734 & 41.4289 & & & \\
\hline $\operatorname{ARIMA}(1,0,1)$ & 7.747 & 35.300 & $\operatorname{ARIMA}(3,0,4)$ & 6.947 & 43.8065 & & & \\
\hline $\operatorname{ARIMA}(1,0,2)$ & 7.771 & 37.177 & $\operatorname{ARIMA}(3,0,5)$ & 6.631 & 45.2426 & & & \\
\hline $\operatorname{ARIMA}(1,0,3)$ & 7.441 & 38.644 & $\operatorname{ARIMA}(4,0,1)$ & 7.139 & 40.1383 & & & \\
\hline
\end{tabular}

3 Smoothing using smooth exponential series

After smoothing data using exponential smoothing series, it has been found that ARIMA $(1,0,0)$ was the best model because it has the minimum values in MSE and $\operatorname{AIC}(\mathrm{k})$ models.

\begin{tabular}{|c|c|c|c|c|c|c|c|c|}
\hline Model & MSE & AIC(k) & Model & MSE & AIC(k) & Model & MSE & AIC) \\
\hline ARIMA(0,0,1) & 105.45 & 65.840 & ARIMA(1,0,4) & 38.63 & 60.530 & ARIMA(4,0,1) & 32.441 & 58.25 \\
\hline ARIMA(0,0,2) & 55.05 & 59.200 & ARIMA(1,0,5) & 35.399 & 61.390 & ARIMA(4,0,2) & 35.457 & 61.41 \\
\hline ARIMA(0,0,3) & 41.25 & 57.400 & ARIMA(2,0,1) & 33.784 & 54.780 & ARIMA $(4,0,3)$ & 31.682 & 61.90 \\
\hline ARIMA(0,0,4) & 41.55 & 59.490 & ARIMA(2,0,2) & 32.247 & 56.170 & ARIMA(4,0,4) & 29.15 & 62.80 \\
\hline ARIMA(0,0,5) & 43.04 & 61.960 & ARIMA(2,0,3) & 38.71 & 60.000 & ARIMA(4,0,5) & 31.925 & 66.04 \\
\hline ARIMA(1,0,0) & 37.45 & 52.130 & ARIMA(2,0,4) & 36.214 & 61.000 & ARIMA(5,0,1) & 33.821 & 60.79 \\
\hline ARIMA(2,0,0) & 36.1 & 53.650 & ARIMA(2,0,5) & 34.271 & 62.970 & ARIMA(5,0,2) & 28.388 & 60.51 \\
\hline ARIMA(3,0,0) & 37.09 & 56.005 & ARIMA(3,0,1) & 33.952 & 56.840 & ARIMA(5,0,3) & 33.759 & 64.77 \\
\hline ARIMA(4,0,0) & 38.04 & 58.330 & ARIMA(3,0,2) & ------ & $*$ & ARIMA(5,0,4) & 44.111 & 70.28 \\
\hline ARIMA(5,0,0) & 34.181 & 58.930 & ARIMA(3,0,3) & 27.965 & 58.310 & & & \\
\hline ARIMA(1,0,1) & 36.74 & 53.880 & ARIMA(3,0,4) & 34.547 & 63.070 & & & \\
\hline ARIMA(1,0,2) & ------- & & ARIMA(3,0,5) & 29.625 & 63.068 & & & \\
\hline ARIMA(1,0,3) & ------- & & & & & & \\
\hline
\end{tabular}

\section{Conclusions:}

The paper results have shown that the proposed rainfall series was stationary in its performance. While the beast achieved sample for rainfall was for autoregressive- moving average models ARIMA $(5,0,2)>$ that can be interpreted by the minimum values in both AIC (K) and MSE for this model. However, after transformation, by square root method, ARIMA $(1,0,0)$ offers the best smooth, but ARIMA $(1,0,0)$ was the best when smoothing data using smooth exponential series. Thereby, and by comparing the obtained results, it can be concluded that a significant improvement has occurred in the model after transformation and smoothing. Therefore, it is recommended to use the transformation approach and the methods of exponential smoothing to obtain the best prediction model.

\section{References}

1. Chatfiled, C.( 1975).." The Analysis of time Series Theory and practice" Chaman and Hdi, London.

2. Douglas, N,"Bayesian Confidence Intervals for Smoothing splines " JASA-DECEMBER , 1988,v83 N. 404.

3. James, W.Taylor (2003)" Exponential Smoothing with a damped Multiplicative Trend " International Journal of Forcasting" Vol.19, pp 715-725.

4. James, W.Taylor (2004)" Volatility Forcasting with Smooth Transition Exponential Smoothing " International Journal of " Vol.20 ,pp 273-286. 
5. Spyros, M, Steven .C.W\&Victor E.M(1983);"Forecasting Methods and Application"second Edition,John wiley\&Sons.

6. Stanly, L.Sclove (2002)" Exponential Smoothing and Box-Jenkis ARIMA Models" ILLinois Chicago.

7. Priestley, M. B., (1981) "SPECTRAL Analysis and Time Series", Vol. 1, Department of Mathematics, University of Manchester, Academic Press Inc. London, UK.

8. Voind, H.D.(1999)"Time Series analysis" Economic Fordham University, Bronex, New York, USA.

9. Yogesh Hole et al 2019 J. Phys.: Conf. Ser. 1362012121 